\title{
NIVEL DE CONOCIMIENTO, USO Y APLICABILIDAD DE LA TECNOLOGÍA POR PARTE DE LOS EDUCADORES EN EL DESARROLLO DE LA LECTURA DE ALUMNOS CON PROBLEMAS ESPECÍFICOS DE APRENDIZAJE
}

\author{
José Antonio Pérez Rivera \\ Universidad Internacional Iberoamericana (Puerto Rico) \\ jperezrivera@yahoo.com
}

\begin{abstract}
Resumen. Introducción: El objetivo de esta investigación ha sido determinar el nivel de conocimiento de los educadores con respecto al uso de la tecnología y la frecuencia de su aplicabilidad en el proceso de enseñanza y aprendizaje de los alumnos con problemas de aprendizaje en el desarrollo de la lectura. Metodología: Esta investigación ha seguido una metodología cuantitativo, no experimental, correlacional con una muestra de 150 maestros del programa de español en las escuelas públicas de las Regiones de Ponce y Guayama, Puerto Rico, seleccionada por estratificado al azar. Estos respondieron a un cuestionario con una escala tipo Likert sobre Conocimiento y Aplicación de las TIC en el Proceso Lector (CSCA-TIC-PL). Resultados: Se encontró un conocimiento alto-moderado del uso por parte de los maestros, una aplicabilidad promedio-bajo en la sala de clases y resultados moderado-altos, según su percepción. Las variables de estudio estaban correlacionadas entre sí. Discusión: A mayor uso de la tecnología mayor resultado en el desarrollo de destrezas lectoras en estudiantes con necesidades específicas de aprendizaje. Se recomienda ofrecer talleres a maestros sobre la importancia de esta estrategia en la sala de clases.
\end{abstract}

Palabras clave: conocimiento, aplicabilidad, alumnos, lectura, tecnología

\section{LEVEL OF KNOWLEDGE, USE AND APPLICABILITY OF THE TECHNOLOGY BY EDUCATORS IN THE DEVELOPMENT OF THE READING OF STUDENTS WITH SPECIFIC LEARNING PROBLEMS}

\begin{abstract}
Introduction: The objective of this research has been to determine the level of knowledge of educators regarding the use of technology and the frequency of its applicability in the teaching and learning process of students with learning problems in the development of reading. Methodology: This research has followed a quantitative methodology, correlational with a sample of 150 teachers of the Spanish program in the public schools of the Regions of Ponce and Guayama, Puerto Rico, selected by random stratification. They responded to a questionnaire with a Likert scale on Knowledge and Application of ICT in the Reader Process (CSCA-TIC-PL). Results: Them show a high-moderate knowledge of the use by the teachers; a low-average applicability in the classroom, and high-average results, according to their perception. The study variables were correlated with each other. Discussion: The greater the use of technology, the greater the result in the development of reading skills in students with specific learning needs. It is recommended to offer workshops to teachers on the importance of this strategy in the classroom.
\end{abstract}

Keywords: knowledge, applicability, students, reading, technology 


\title{
NÍVEL DE CONHECIMENTO, USO E APLICABILIDADE DA TECNOLOGIA PELOS EDUCADORES NO DESENVOLVIMENTO DA LEITURA DE ALUNOS COM PROBLEMAS ESPECÍFICOS DE APRENDIZAGEM
}

\begin{abstract}
Resumo. Introdução: O objetivo deste estudo foi determinar o nível de conhecimento dos educadores em relação ao uso de tecnologia e a frequência de sua aplicabilidade no processo de ensino e aprendizagem de alunos com problemas de aprendizagem no desenvolvimento da leitura. Metodologia: Este estudo seguiu uma metodologia quantitativo, não experimental, correlacional com uma amostra de 150 professores do programa de espanhol nas escolas públicas das Regiões de Ponce e Guayama, Porto Rico, selecionados por estratificação aleatória. Eles responderam a um questionário com uma escala Likert sobre Conhecimento e Aplicação de TIC no Processo Reader (CSCA-TIC-PL). Resultados: Encontrou-se um alto-mderado conhecimento do uso pelos professores, uma aplicabilidade média-baixa na sala de aula e resultados moderado-alto, de acordo com sua percepção. As variáveis do estudo foram correlacionadas entre si. Discussão: Quanto maior o uso de tecnologia, maior o resultado no desenvolvimento de habilidades de leitura em alunos com necessidades específicas de aprendizagem. Recomenda-se oferecer oficinas aos professores sobre a importância dessa estratégia na sala de aula.
\end{abstract}

Palavras-chave: conhecimento, aplicabilidade, alunos, leitura, tecnologia

\section{Introducción}

Las nuevas corrientes educativas se basan cada vez más en el uso de tecnologías en el proceso de enseñanza y aprendizaje. Esto debido a que la computadora es considerada como una herramienta eficaz en el procesamiento y análisis de datos que ayudan a su fácil manejo. Las escuelas y centros educativos deben establecer planes estratégicos que vayan destinados al desarrollo del conocimiento sobre su uso y aplicabilidad en la sala de clases por parte de los profesores, con la finalidad de ayudar a los alumnos a alcanzar las metas propias y de las comunidades en las que se desenvuelven (De Jesús, 2011; Mirabal, 2016). Además, de estar fundamentadas en las dos justificaciones para el uso de esta: el sentido común generado por las creencias populares y la investigación educativa (Ferrer, 2015).

Para Mirabal (2016), las escuelas deben servir como centros de aprendizaje que apoyen el proceso tecnológico. La educación del siglo XXI propuesta en Puerto Rico, debe estar inmersa en las nuevas corrientes de tecnología educativa. Por tanto, la misma debe ofrecer oportunidades dentro de un ambiente de aprendizaje formal, pero que a su vez no solo contemple los espacios físicos y los medios, sino también los elementos básicos del diseño instruccional (De Jesús, 2011). Para que esto es necesario un cambio de paradigma donde los educadores tomen parte activa en el uso de las nuevas tecnologías de comunicación e innovación y las incluyan en sus planes de enseñanza de distintas destrezas y habilidades cognitivas (Salcito, 2017).

La lectura es parte de este proceso de aprendizaje tecnológico. De acuerdo con Aguillón (2013), aprender a leer mal desde niños afecta la comprensión de textos de las personas a lo largo de su vida. Esto coincide con las ideas expuestas por López (2016b) quien señaló que los resultados obtenidos en pruebas estandarizadas, como lo son las pruebas administradas por el Programa para la Evaluación Internacional de Alumnos (PISA), se ven reflejadas con el paso del tiempo en los resultados de los alumnos en las universidades. Aspecto que es señalado, de igual manera por De Jesús (2017), quien señala que los procesos de aprendizaje lector deben ser aplicados desde la niñez temprana para que la vida de los individuos sea una objetiva en la etapa adulta. 
En Puerto Rico, los estudiantes con problemas de comprensión lectora son clasificados como Estudiantes con Problemas Específicos de Aprendizaje (Santiago, 2017). La Ley IDEA, Individuals with Disabilities Education Improvement Act (United States Department of Education, 2004), se conoce en español como el Acta para el Mejoramiento de la Educación de Individuos con Impedimentos, define estos problemas como aquellos desordenes que involucran dificultad en entender o en usar el lenguaje hablado o escrito y que se pueden manifestarse en dificultad para escuchar, pensar, hablar, leer, escribir, deletrear o hacer cálculos matemáticos. También, señala que los mismos no deben ser consecuencia de impedimentos visuales, auditivos, motrices, retardo mental (impedimento cognitivo), disturbios emocionales, desventaja económica, cultural o ambiental.

Las leyes que cobijan y hacen extensiva los servicios que se ofrecen en el Programa de Educación Especial en Puerto Rico son la Ley 149 del 30 de junio de 1999: Ley Orgánica del Departamento de Educación de Puerto Rico (Estado Libre Asociado de Puerto Rico, 1999) y; Ley Estatal 51 de 1996: Ley de Servicios Educativos Integrales para Personas con Impedimentos (Estado Libre Asociado de Puerto Rico, 1996). Además, las resoluciones, órdenes y estipulaciones judiciales en el caso Rosa Lydia Vélez forman parte de la reglamentación vigente relacionada con la manera en que se brindan dichos servicios a los estudiantes puertorriqueños (Santiago, 2017). La Carta Circular 9-2016-2017: Política Pública Sobre la Organización Escolar y Requisitos de Graduación de las Escuelas de la Comunidad Elementales y Secundarias del Departamento de Educación de Puerto Rico; estableció el uso de la tecnología como una estrategia para servir a la población con impedimentos y que debe ser garantizada en la planificación escolar.

Pero, uno de los mayores retos es la visión que tienen los educadores sobre este proceso. El primero problema es que la enseñanza de la comprensión lectora se confunde con otras destrezas de comprensión. Debido a esto, los maestros se enfocan más en la exposición repetitiva donde se lee, se contestan preguntas y se corrigen aquellas respuestas incorrectas. No existen los procesos de análisis del significado global y la inferencia como consecuencia de este (Varela, 2018).

López (2016b), cuando hace referencia al poco desempeño de los alumnos, tanto a nivel internacional como nacional, en las pruebas PISA, recomienda el analizar la manera en cómo se está enseñando en sistema público y privado de la Isla. Esto debido a que los resultados en esta prueba evidencian que ninguno de los dos sistemas está a un alto nivel internacional. Esta recalca que no se trata de los currículos que se utilizan, sino del sentido que se le da a la enseñanza en sí misma, por tanto, es importante repensar el modelo educativo a implementarse en la sala de clases.

Esta situación se agrava cuando las estadísticas en Puerto Rico señalan que el $20 \%$ de la población tiene algún tipo de impedimento de un total de 3,725,789 de puertorriqueños. Para el año escolar 2016-2017, existían unos 146,985 estudiantes de educación especial que representan el $39 \%$ de la matricula total de alumnos en el sistema educativo. De estos aproximadamente el $70 \%$ estaba recibiendo servicios de inclusión en la sala de clases regular. De esta matricula, el 50.4\% de los alumnos presentaban deficiencias en las destrezas lectoras entre otras deficiencias mucho más agudas en el desarrollo cognoscitivo (López, 2016a; López, 2016b, Santiago, 2017).

Por otro lado, la mayoría de los educadores ven a los estudiantes como un ente receptivo pasivo, mientras el maestro es quien posee todo el conocimiento. Esto lleva a que los segundos no desarrollen la crítica, la reflexión, la independencia, la creatividad y la responsabilidad cotidiana. La educación es vista como un proceso que no debe salirse de lo tradicional y se olvida que la aptitud y el interés de los alumnos para 
aprender deben ser desarrollados paralelamente. Por tanto, se debe diversificar los programas curriculares de acuerdo con la realidad de las necesidades presentes (Varela, 2018; Santiago, 2017). Estos datos hacen imprescindible la necesidad de estudiar los beneficios de las nuevas tecnologías en el desarrollo de la lectura (Mirabal, 2016).

Al establecer como trasfondo las ideas de De Jesús (2011) y Mirabal (2016), esta investigación tuvo el objetivo de determinar cuáles eran las expectativas de los educadores con respecto al uso de la tecnología en el proceso de enseñanza y aprendizaje de los alumnos con problemas de aprendizaje en el desarrollo de la lectura. La misma se enfocó, específicamente, en las necesidades académicas de los estudiantes, así como el ambiente, las condiciones pedagógicas y contextuales. Además, se consideró el conocimiento y sus relaciones con los individuos como el factor principal para formar una sociedad del conocimiento (De Jesús, 2011). Pues, tal como señalaba Mirabal (2016) y Formichella y Aldarete (2018), las nuevas corrientes tecnológicas en los ambientes educativos deben atender los distintos componentes que constituyen la vida escolar y la inmersión de los alumnos en la sociedad fuera de estos ambientes. Por esta razón, esta investigación va dirigida a estudiar la utilización de la computadora en el proceso enseñanza y aprendizaje, realzando el objetivo de apuntar un complemento atractivo para su utilización.

Esta investigación permite establecer un antecedente para la planificación efectiva por parte de las escuelas para ofrecer talleres o adiestramientos referentes al uso de los recursos tecnológicos como estrategia de enseñanza. También, aporta a la búsqueda de medios más costo efectivos que puedan revertir los bajos índices en el desarrollo de las destrezas de comprensión lectora. Esto para mejorar la calidad del sistema y los servicios que se ofrecen a los estudiantes. Asunto que debe ser considerado como primordial cuando se trabaja con estudiantes con necesidades especiales, según Varela (2018).

Como marco conceptual se utilizan las investigaciones recientes sobre el tema. Por ejemplo, Herrera (2010) realizó un estudio cuasi experimental con estudiantes de básica primaria donde encontró que alguno de estos se movieron de una actitud contemplativa a un rol más participativo en el que mostraron interés por lo aprendido, así como un aumento en la capacidad para interpretar y analizar que se tradujo en una mayor capacidad de interpelación, critica frente a los contenidos académicos y una mayor elaboración de deducciones y conceptualizaciones. Según este, la incorporación de la tecnología en el proceso de enseñanza y aprendizaje lector promueve un cambio de actitud notable frente a la lectura de diversos textos y/o documentos facilitando así las habilidades que se esperan en el nivel educativo de los alumnos.

Por su parte, Hernández y Arteaga (2011) realizaron un estudio cualitativo con estudiantes de grado primario quienes fueron sometidos a un tratamiento de actividades lectoras por un periodo de tres semanas utilizando nueva tecnología. Los resultados reflejaron que el procesamiento lector estaba presente cuando los alumnos interactuaban con un texto experto enfocado a cuestionar sobre los significados y sentidos. Los participantes demostraron que esta destreza puede alcanzarse desde los niveles de anticipación e inferencia, por lo que es necesario centrar interés en las prácticas de interacción textual con la intención de anticipar las reacciones de los alumnos a los textos, en especial a los simbolismos presentes en la lectura. Sin embargo, según los investigadores, es difícil encontrar material didáctico de manera tecnológica que ayude con el proceso de comprensión lectora por lo que se hace imprescindible la intervención constante del docente en la planificación como en la práctica, pues el solo uso de la herramienta no garantiza el aprendizaje. 
Aburto (2011) realizó un estudio exploratorio en donde 20 educadores respondieron preguntas sobre la utilidad de la tecnología en la sala de clases. Estos demostraron estar conscientes de la importancia de la TIC en la Educación Especial y reconocen haber cambiado sus prácticas docentes al utilizar recursos tecnológicos, pues estos resultan valiosos e importantes. El investigador señala que, a pesar de la introducción aparentemente efectiva de esta herramienta, no existe una aplicabilidad adecuada que permita maximizar los resultados a favor de los alumnos, por lo que se hace necesario esfuerzos adicionales en el adiestramiento docente que permita el uso adecuado de las mismas.

Por su parte, Samaniego, Laitamo, Valerio y Francisco (2012) realizaron un estudio documental sobre la inclusión de la tecnología en los procesos educativos en el Caribe. El mismo permitió destacar la ausencia de políticas específicas relativas a las TIC a las personas con necesidades especiales, donde solo $50 \%$ de los países estudiados habían firmado acuerdos para garantizar el uso de las mismas en la educación de esta población. Estos recomiendan el promover el intercambio y la incorporación de las nuevas tecnologías de información y comunicación dentro de los planes curriculares de educación con el objetivo de promover a todos los niveles el conocimiento. Finalmente, señalan la necesidad de educar a los profesionales con respeto a la utilidad de estos recursos, en especial para la formación de personas con necesidades especiales en esta región.

Abbruzzese (2018), realizó un estudio longitudinal para establecer los patrones de uso tecnológico en la Educación Especial. Los resultados demostraron altos niveles de aceptabilidad de este tipo de recurso en las escuelas. Además, se encontró que las mismas son beneficiosas en el proceso de aprendizaje de estudiantes con necesidades especiales, logrando que estos se sientan incluidos a través de las metodologías innovadoras, esto a pesar de que los maestros no identifiquen su beneficio directamente. La investigadora señala que este tipo de herramienta es necesaria para satisfacer las necesidades de la población estudiada, pues le permite integrarse a la corriente regular de alumnos de una forma adecuada en la que se consideran sus necesidades y potencialidades.

Fernández, Rodríguez, Rodríguez y Martínez (2013) realizaron un estudio cuasi experimental con 39 estudiantes españoles para determinar la efectividad de las TIC en la educación. Estos encontraron que el uso de la misma se asocia con efectos positivos en el desarrollo de habilidades de aprendizaje, observando que se mejoraron las destrezas básicas de lenguaje, matemáticas, conciencia ambiental, autonomía y social. Además, se encontró que, en muchos casos, los alumnos tuvieron la oportunidad de realizar actividades que antes no le eran accesibles. Los investigadores sugirieron que el repertorio de tipos de actividades debe ser proporcionado de acuerdo a los propósitos de aprendizaje de los estudiantes con impedimentos o necesidades extraordinarias.

Teóricamente es necesario tener en cuenta que el aprendizaje es una experiencia sociocultural, de acuerdo con Lev S. Vygotsky (1978), donde el ambiente en el que se desarrollan los estudiantes es fundamental para que se activen las acciones correctas del conocimiento. Además, es importante entender que este proceso debe ser uno por descubrimiento, tal como señalaba Jerome Bruner (1985), para que sea pertinente a las necesidades el alumno y las destrezas se puedan arraigar en la memoria de este. Finalmente, se debe considerar que en la actualidad las nuevas tecnologías con parte esencial de la vida, por lo que la conectividad es esencial en los procesos para que sean útil, según lo especificaba George Siemens (2006) en sus postulados. En la Figura 1 se puede observar la manera en que los componentes actúan entre sí para que el proceso de enseñanza y aprendizaje sea completado de manera asertiva. 


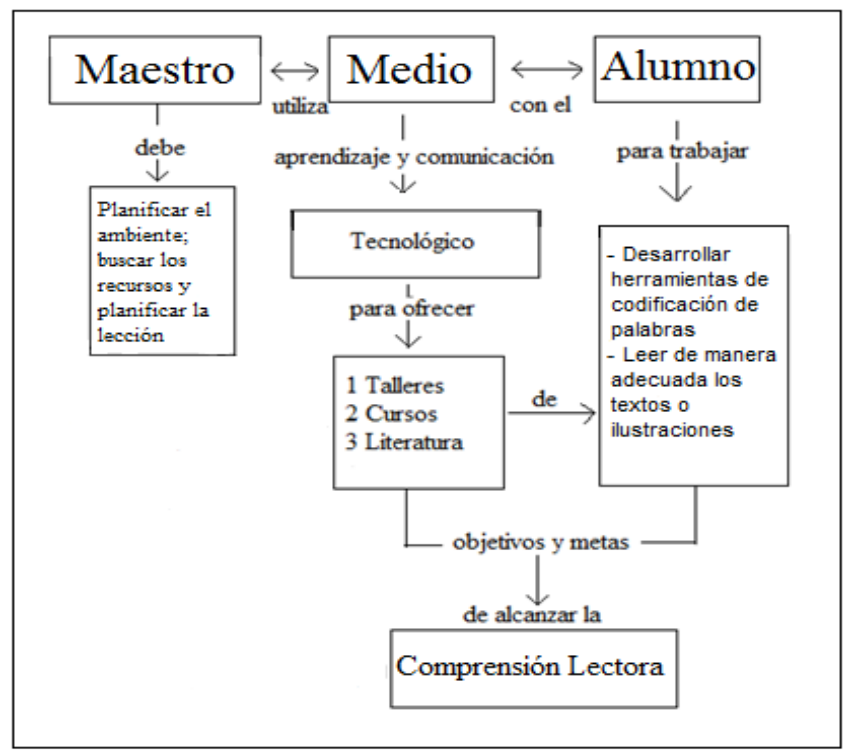

Figura 1. Proceso enseñanza-aprendizaje con TIC (De Jesús, 2011).

\section{Método}

Esta investigación siguió un diseño no experimental con enfoque cuantitativo. La población, durante el año escolar 2017-2018, se estimó en 1,272 escuelas públicas en el país adscritas al Departamento de Educación de Puerto Rico y con una matrícula de maestros contratados de 20,915 en todas las materias y niveles educacionales (Departamento de Educación de Puerto Rico, 2018). La muestra de esta investigación estuvo compuesta por maestros del programa de español en las escuelas públicas de las Regiones de Ponce y Guayama. Para el muestreo se utilizaron márgenes absolutos para poblaciones infinitas. Se utilizaron los siguientes datos, recomendados por Herrera (2011) para el número final muestral: Población de 267; Probabilidad de 0.50; Confiabilidad de 0.95 y Margen de Error de 0.05. La muestra total fue de 150 lo que representaba el $56 \%$ de estos.

Como parte del proceso investigativo, se obtuvieron las autorizaciones del Departamento de Educación de Puerto Rico y la Junta de Revisión Institucional de la Universidad Internacional Iberoamericana, recinto en línea de Cataluña, España. Se coordinó y visitó las escuelas para ser orientadas sobre los propósitos y derechos y responsabilidades de participación. Se administraron los instrumentos y se realizarán los análisis estadísticos correspondientes. Todos los procesos fueron anónimos y no representaban daño alguno a los participantes. Los hallazgos fueron analizados y contractados con investigaciones previas, tal como lo recomienda De Jesús (2018).

Para la recolección de los datos se utilizó el Cuestionario Sobre Conocimiento y Aplicación de las TIC en el Proceso de Lector (CSCA-TIC-PL) con cuatro secciones separadas. La primera recopiló un perfil sociodemográfico de los participantes. La segunda era una escala Likert de cuatro puntos (Totalmente de acuerdo - Totalmente en desacuerdo) que media el conocimiento respecto al uso de la tecnología como estrategia educativa y con una escala valorativa de alto, moderado, bajo o ningún conocimiento. La tercera era una escala Likert de cuatro niveles de ejecución (Siempre - Nunca) que media la implementación de la estrategia en la sala de clase y con una escala valorativa de excelente, superior, promedio, bajo promedio y deficiente aplicabilidad. La cuarta era una escala Likert de cuatro puntos (Totalmente de acuerdo - Totalmente en desacuerdo) que media la percepción de los educadores con respecto al 
aprovechamiento académico de los alumnos relacionado al proceso lector y con una escala valorativa de alto, moderado, bajo o ningún aprovechamiento. Todos los reactivos fueron redactados basándose en la revisión de constructos.

El cuestionario se sometió a juicio de un panel de expertos para determinar su constructo. Para determinar su confiabilidad interna, se utilizó la muestra total de los participantes de este estudio, esto para hacerlo útil a futuras investigaciones, y se obtuvo un coeficiente de Alpha de Cronbach de $\mathrm{C} \alpha=.808$ en el cuestionario de conocimiento; $\mathrm{C} \alpha=.942$ en el cuestionario sobre aplicabilidad y $\mathrm{C} \alpha=.957$ en el cuestionario sobre percepción del aprovechamiento académico. Estos resultados representan una confiabilidad alta para instrumentos de este tipo. Para los análisis de los hallazgos se utilizó estadística descriptiva de frecuencia y por ciento, medidas de tendencia central (mediana y moda) y correlación de Pearson (r) para establecer la relación entre las variables.

\section{Resultados}

El perfil sociodemográfico reflejó que el $86 \%$ de los participantes de esta investigación eran del sexo femenino y el $14 \%$ masculino con una edad media entre los 41 y 55 años. El $60 \%$ poseía un grado de bachillerato, el $37 \%$ de maestría y el $3 \%$ de doctorado en el campo laboral. El 72\% de estos cumplían funciones como maestros de la sala regular, mientras que el $28 \%$ eran maestros del salón recurso. Estos, en su mayoría, tenían más de 10 años de experiencia.

El 51\% de los maestros participantes señaló haber recibido algún tipo de adiestramiento previo en el uso de TIC como estrategia de enseñanza para estudiantes con necesidades especiales. El 49\% respondió en la negativa. Estos resultados sugieren que la mitad de los profesionales que deben manejar este tipo de herramienta didáctica están adiestrados. Estos resultados tienen un margen de error de .041, con una desviación estándar de .501 lo que significa que son confiables (Tabla 1).

Tabla 1

Maestros con adiestramientos previos)

\begin{tabular}{|c|c|c|c|}
\hline $\begin{array}{c}\text { ¿Has recibido adiestramiento anteriormente } \\
\text { sobre el uso de las Tecnologías de } \\
\text { Información y Comunicación? }\end{array}$ & $f$ & $\%$ & media \\
\hline Sí & 77 & 51 & .514 \\
\hline No & 73 & 49 & .486 \\
\hline Total & 150 & 100 & 1.000 \\
\hline
\end{tabular}

El nivel de conocimiento de los maestros sobre esta estrategia educativa se puede considerar como uno alto-moderado, donde el 65\% de ellos demostró niveles significativos. El $64.67 \%$ demostró un nivel alto en esta categoría, mientras que un $35.33 \%$ demostró niveles moderados. Ninguno de los educadores obtuvo niveles bajos en este renglón. Estos resultados tienen un margen de error de .59 y una desviación estándar de 7.25; aunque exista una desviación de siete (7) puntos negativos, los resultados continúan siendo altos para el nivel de conocimiento, lo que se puede interpretar como una constante para esta variable (Tabla 2).

En los resultados detallados del CSCA-TIC-PL se encontró que los maestros conocen los propósitos del uso de la tecnología como herramienta de apoyo para 
estudiantes. Estos, además, están conscientes que, en el caso de estudiantes con necesidades especiales, las TIC son un recurso adicional que permite el avance de los mismos. También, están conscientes de que ellos, como educadores, deben estar preparados profesionalmente en la materia que enseñarán para poder maximizar este recurso didáctico.

Sin embargo, se puede observar cuatro (4) aspectos en los que los maestros necesitan apoyo. Estos son: 1) Entender que el uso de las TIC es un recurso de apoyo para estudiantes con necesidades especiales; 2) que este tipo de recurso no solo se debe usar en la sala de salón recurso, sino que puede ser usado en las salas regulares de clase; 3) que se puede usar técnicas jerárquicas y de cooperación como parte del proceso de enseñanza y aprendizaje y; 4) que este tipo de recurso ha demostrado ser efectivo en estudios anteriores.

Tabla 2

Nivel de conocimiento respecto al uso

\begin{tabular}{lcc}
\hline \multicolumn{1}{r}{ Nivel de conocimiento } & $f$ & $\%$ \\
\hline Alto & 97 & 64.67 \\
Moderado & 53 & 35.33 \\
Bajo & 0 & 0.00 \\
Ninguno & 0 & 0.00 \\
$\quad$ Total & 150 & 100.00 \\
\hline
\end{tabular}

El nivel de ejecución referente al uso de la misma como parte de las herramientas de enseñanza fue de $77 \%$ lo que es un uso promedio-bajo. El $50.67 \%$ de los maestros demostró niveles promedios aplicabilidad de las TIC en sus lecciones para el desarrollo lector. El $26 \%$ fue bajo promedio y el $10.67 \%$ fue superior. Un $7.32 \%$ obtuvo niveles deficientes, mientras que solo el $5.34 \%$ de estos demostró aplicar las mismas en un nivel de excelencia. Estos resultados tienen un margen de error de 1.16 y una desviación estándar de 14.19; esta desviación de 14 puntos negativos puede reflejar resultados distintos para el nivel de aplicabilidad, lo que se puede interpretar como una variable poco constante (Tabla 3).

En los datos detallados del CSCA-TIC-PL se encontró que los maestros tienden a reconocer a aquellos alumnos que alcanzan las metas antes, durante y después del uso de las TIC como herramienta didáctica. Además, estos identifican las destrezas a ser desarrolladas, tanto generales como individuales, de los estudiantes con la finalidad de incluirlas en los planes de lección. Sin embargo, entienden que no existe un apoyo por parte de los coordinadores del Programa de Educación Especial en cuanto al uso de estas en la sala de clases. La mayoría de ellos, también, señaló que en los Planes Educativos Individualizados no aparece como recurso viable y que los padres demuestran poco o ningún interés con respecto al uso como alternativa de enseñanza. 
Tabla 3

Nivel de aplicabilidad de TIC

\begin{tabular}{lcc}
\hline \multicolumn{1}{c}{ Nivel de aplicabilidad } & $f$ & $\%$ \\
\hline Excelente & 8 & 5.34 \\
Superior & 16 & 10.67 \\
Promedio & 76 & 50.67 \\
Bajo promedio & 39 & 26.00 \\
Deficiente & 11 & 7.32 \\
\multicolumn{1}{c}{ Total } & 150 & 100.00 \\
\hline
\end{tabular}

En relación con la percepción de los maestros referente al aprovechamiento académico de los estudiantes después de la utilización se puede considerar uno moderado-alto, pues la mayoría de estos señaló que sus pupilos obtuvieron resultados positivos en el desarrollo de destrezas lectoras. El $56 \%$ de los maestros piensa que sus estudiantes obtuvieron resultados moderados después de haber aplicado esta herramienta en la sala de clases. El $42.67 \%$ piensa que ese aprovechamiento tiene un nivel alto, mientras que solo el $1.33 \%$ lo ubica como bajo. Estos resultados tienen un margen de un error de .76 y una desviación estándar de 9.37; esta desviación de nueve (9) puntos negativos se resumen en que los datos podrían reflejar resultados distintos para el nivel de aprovechamiento, lo que se puede interpretar como una variable poco constante (Tabla 4).

En los datos detallados del CSCA-TIC-PL, se encontró que, como parte del aprovechamiento académico y consecuencia del uso de las TIC con estudiante con necesidades especiales en la comprensión lectora, estos tuvieron alcances sustanciales. Por ejemplo, estos lograron hacer predicciones y anticipar contenidos del texto, así como identificar hechos y opiniones en los mismos. Además, lograron desarrollar habilidades para la transferencia de información de un texto a otro, según la percepción de los maestros.

Por otro lado, las tres (3) dificultades de los estudiantes, según los maestros, después del uso de las TIC fueron: 1) Estos no lograron encontrar elementos de cohesión a las oraciones y párrafos que configuraban los textos; 2) estos no lograron interpretar un texto sin entenderlo del todo y; 3) estos no desarrollaron las habilidades para transferir información de un texto a otro.

Tabla 4

Nivel de aprovechamiento académico

\begin{tabular}{lcc}
\hline \multicolumn{1}{r}{ Nivel aprovechamiento } & $f$ & $\%$ \\
\hline Alto & 64 & 42.67 \\
Moderado & 84 & 56.00 \\
Bajo & 2 & 1.33 \\
Ninguno & 0 & 0.00 \\
$\quad$ Total & 150 & 100.00 \\
\hline
\end{tabular}


En relación con las correlaciones entre variables, los resultados reflejaron que estas tienen una significancia positiva baja. Entre el conocimiento de los maestros y la aplicabilidad la correlación de Pearson fue de .369, mientras que con el aprovechamiento académico de los estudiantes fue de $.265 \mathrm{y}$, entre la aplicabilidad y el aprovechamiento fue de .552. Estas fueron a margen de error de .05. Esto significa que, si una de las variables sube en el espectro de onda, la otra subirá en la misma correspondencia (Tabla 5).

Tabla 5

Análisis de correlación Pearson

\begin{tabular}{lllll}
\hline & & $(1)$ & $(2)$ & $(3)$ \\
Conocimiento & Pearson $(r)$ & 1 & $.369^{* *}$ & $.265^{* *}$ \\
& Significancia & & .000 & .001 \\
& Muestra & 150 & 150 & 150 \\
& Pearson $(r)$ & $.369^{* *}$ & 1 & $.552^{* *}$ \\
Aplicabilidad & Significancia & .000 & & .000 \\
& Muestra & 150 & 150 & 150 \\
Aprovechamiento & Significancia & $.265^{* *}$ & $.552^{* *}$ & 1 \\
& Muestra & .001 & .000 & \\
& Pearson $(r)$ & 150 & 150 & 150 \\
\hline Nota:**Correlación es a una significancia de nivel 0.01 (2-rabos) & & &
\end{tabular}

Nota: $* *$ Correlación es a una significancia de nivel 0.01 (2-rabos)

\section{Discusión y conclusiones}

Esta investigación llega a las siguientes conclusiones: Primero, el nivel de conocimiento de los educadores con respecto al uso de la tecnología en el proceso de enseñanza y aprendizaje es alto-moderado. Estos resultados concuerdan con los encontrados por Cozar, De Moya, Hernández y Hernández, (2016) donde la mayoría de los futuros maestros poseen destrezas adecuadas en el uso de la tecnología como herramienta de trabajo y, donde, la mayoría de estos la prefiere como estrategia en la sala de clases. Esto implica que están adiestrados en el uso de la tecnología y que puede ser un factor determinante en su carrera profesional. Sin embargo, se recomienda que los mismos continúen tomando curos o talleres de educación continua, tal como señala Mirabal (2016).

Segundo, el nivel de implementación de la tecnología en el proceso de enseñanza y aprendizaje de los alumnos con problemas de aprendizaje en el desarrollo de la lectura es promedio-bajo. Esto es indicativo de que el sistema cuenta con recursos adecuados, pero no existe la predisposición de los educadores respecto al uso. Se debe orientar a que los mismos consideren esta herramienta de trabajo en sus cursos para beneficio de los estudiantes y para el mejor resultado de las pruebas estandarizadas, tal como recomienda Salcito (2017) cuando sugiere un cambio paradigmático con relación al tema. Además, estos resultados implican, tal como expuso Abbruzzese (2018), la necesidad de crear herramientas que tengan el objetivo de identificar procesos de comprensión lectora. Pues, la tecnología ha demostrado ser útil en la aplicación puesto 
que permite planificar y ejecutar el proceso de enseñanza y aprendizaje debido a facilidad de manipulación por parte de los alumnos.

Es importante mencionar que esta variable obtuvo una desviación de 14 puntos negativos. Esto se resume en que los resultados podrían reflejar resultados distintos para el nivel de aplicabilidad, lo que se puede interpretar como una variable poco constante. Esto podría tener como explicación que la cantidad de recursos tecnológicos disponibles en las escuelas públicas de Puerto Rico son escasos y no precisamente por el factor de desconocimiento, tal como señala De Jesús (2011) y que concuerda con los señalamientos de Mirabal (2016). Esta última señala la importancia de establecer recursos económicos que ayuden a la obtención de herramientas tecnológicas para hacerlas accesibles a todos los maestros y estudiantes por igual.

Tercero, el nivel de aprovechamiento académico de los estudiantes luego de la implementación de la tecnología como estrategia para ayudarlos en el desarrollo de las habilidades y destrezas lectoras, según la percepción de los maestros, es moderado-alto. Esto es como resultado de la implementación promedio de la tecnología como estrategia de enseñanza y aprendizaje, Varela (2018) señalaba que el uso de estrategias diversas ayuda a mantener los estándares y promedios en las pruebas de lectura y destrezas relacionadas con el lenguaje en esta población con necesidades. Esto concuerda con Formichella y Alderete (2018) quien señalaba que la incorporación de la tecnología en el proceso de enseñanza y aprendizaje lector promueve un cambio de actitud notable frente a la lectura de diversos textos y/o documentos facilitando así las habilidades que se esperan en el nivel educativo de los alumnos y el cual podría ser maximizado en el hogar al utilizar estas herramientas como extensión a la sala de clases.

Esta variable obtuvo una desviación de nueve puntos negativos. Esto se resume en que los resultados podrían reflejar resultados distintos para el nivel de aprovechamiento por parte de los estudiantes, lo que se puede interpretar como una variable poco constante. Ahora bien, esta disminución del nivel se podría explicar en relación con la aplicabilidad, pues a poca aplicabilidad poco aprovechamiento y viceversa (Mirabal, 2016).

Cuarto, existe relación significativa entre las variables estudiadas. Esto significa que en la manera en que una de ellas sube en el espectro de onda la otra subirá en la misma proporción. Esto implica que si se realizan esfuerzos adicionales para incluir la tecnología en el desarrollo de destrezas estas tendrán un resultado positivo en la adquisición del conocimiento y, por ende, en los resultados esperados en las pruebas estandarizadas. Estos resultados podrían tener sesgos debido a que el instrumento es uno auto aplicable. Esto concuerda con Abbruzzese (2018) quien encontró una relación entre la importancia de las TIC en la Educación Especial y las prácticas al utilizar recursos tecnológicos en beneficio de los estudiantes. Sin embargo, tal como demostró su estudio, los educadores tienden a no presentaron evidencia de su utilización en la sala de clases lo que hace necesario que se desarrollen planes para la inclusión de estas como estrategia educativa.

Al aceptar las hipótesis alternas de investigación, se puede inferir la relación que existe entre las variables y, por ende, la necesidad de que se establezcan procedimientos en que el conocimiento de los educadores sea desarrollado para que la aplicabilidad de las TIC y el aprovechamiento de los alumnos se vean positivamente afectados. El éxito dependerá del cambio de paradigmas por parte de los maestros quienes deben replantearse sus estrategias y aportar nuevas propuestas y recursos a la práctica. La capacitación es esencial para el alcance de las metas (Parra, Gámez y Pintor, 2015; Salcito, 2017). 
Por tanto, se recomienda a las agencias encargadas de los Programas de Educación Especial, tanto en Puerto Rico como a nivel internacional, el adiestrar a los maestros respecto a la utilidad de este recurso como estrategia para alcanzar los niveles adecuados según los estándares relacionados a la lectura. Este estudio se limitó a la población de educadores puertorriqueños, por lo que se recomienda replicar el mismo en otras poblaciones. Además, se recomienda a los futuros investigadores el estudiar estas variables en todos los niveles educativos para poder desarrollar pruebas que midan el aprovechamiento académico real de los estudiantes con necesidades específicas de aprendizaje. Esto pues, como señaló De Jesús (2017), es imprescindible cuando se habla de una educación de calidad y excelencia en todos sus aspectos.

Esta investigación permite promover la importancia del desarrollo profesional mediante la utilización de mecanismos más costo efectivo, de modo que se eliminen barreras en la manera en que se trabaja con esta población y se alcanzan los estándares educativos propuestos por los sistemas educativos, tanto en su beneficio como educadores como para los estudiantes que atienden. Además, permite analizar, desde una perspectiva más holística, los niveles de conocimiento que poseen estos sobre la incorporación de las nuevas tecnologías de la información y la comunicación en la sala de clases, así como el provecho académico que se desprende de su utilización como herramienta para el alcance de un mejor aprovechamiento académico por parte de los alumnos con problemas específicos de la comprensión lectora. Finalmente, provee un análisis de situación para la creación de planes de acción para potencializar los recursos humanos y tecnológicos en los Programas de Educación Especial.

\section{Referencias}

Abbruzzese, G. (2018). Una experiencia desde las TIC en la Educación Especial. Tesis de grado, Escuela de Comunicación Social, Universidad Nacional de Rosario, Santa Fe, Argentina.

Aburto, R. (2011). Percepción del uso de TIC en las practicas pedagógicas de los profesores de Educación Especial. Revista Electrónica Educare, 15(2), 163 184. ISSN: 1409-42-58.

Aguillón, C. (2013). Investigación sobre la deficiencia en la lectura y los afectos en la comprensión. Tesis de psicología, Universidad Nacional Autónoma de México (UNAM), México, DF.

Bruner, J. (1985). Child's Talk: Learning to Use Language. New York: W. W. Norton \& Company.

Carta Circular Núm.: 9-2016-2017 del Departamento de Educación de Puerto Rico. (2016). Política pública sobre la organización del Programa de Educación Física en las escuelas elementales y secundarias del Departamento de Educación de Puerto Rico. Hato Rey, PR: Departamento de Educación.

Cozar, R., De Moya, M., Hernández, J. \& Hernández, J. (2016). Conocimiento y uso de las tecnologías de la información y las comunicaciones (TIC) según el estilo de aprendizaje de los futuros maestros. Formación Universitaria, 9(6), 105-118.

De Jesús, N. (2011). La efectividad del e-coaching en el desarrollo profesional y la salud organizacional de los maestros de dos instituciones educativas privadas en la Región Educativa de Ponce, Puerto Rico. Tesis doctoral en Educación, Pontificia Universidad Católica de Puerto Rico, Campus de Ponce.

De Jesús, N. (2017). La educación y los niños. Santa Isabel, PR: Boletín Informativo SoEd. 
De Jesús, N. (2018). Guía básica para la revisión de literatura para proyectos, informes, tesinas, tesis y disertaciones (primera revisión). Tampa, FL: CreateSpace Independent Publishing Platform.

Departamento de Educación de Puerto Rico. (2018). Registro de maestros de español a nivel elemental en las regiones educativas de Ponce y Guayama, Puerto Rico. Entrevista telefónica con los directores regionales, realizada el 14 de febrero de 2018, por el investigador.

Estado Libre Asociado de Puerto Rico. (1996). Ley Estatal 51 de 1996: Ley de Servicios Educativos Integrales para Personas con Impedimentos. Recuperado de, www.lexjuris.com

Estado Libre Asociado de Puerto Rico. (1999). Ley 149 del 30 de junio de 1999: Ley Orgánica del Departamento de Educación de Puerto Rico. Recuperado de, www.lexjuris.com

Fernández, A., Rodríguez, M., Rodríguez, M. \& Martínez, M. (2013). Mobile learning technology based on iOS devices to support students with special education needs. ElSevier, 61, 77-86.

Ferrer, J. (2015). El uso de las microcomputadoras en el salón de clases: El proceso de infusión de la tecnología. Mayagüez: Universidad de Puerto Rico.

Formichella, M. \& Aldarete, M. (2018). TIC en la escuela y rendimiento educativo: el efecto mediador del uso de las TIC en el hogar. Cuaderno Investigación en Educación, 9(1), 75-93.

Hernández, J. \& Arteaga, D. (2011). Las TIC como facilitadoras en la comprensión lectora. Tesis de grado, Facultad de Ciencias Sociales, Humanas y de la Educación Edumática, Universidad Católica de Pereira, Colombia.

Herrera, D. (2010). El proceso de enseñanza-aprendizaje de la comprensión lectora con el uso de las TIC como apoyo pedagógico. Tesis de Licenciatura, Facultad de Educación, Universidad Tecnológica de Pereira, Colombia.

Herrera, M. (2011). Fórmula para cálculo de la muestra poblaciones finitas. Recuperado

de, https://investigacionpediahr.files.wordpress.com/2011/01/formula-paracc3a1lculo-de-la-muestra-poblaciones-finitas-var-categorica.pdf

López, K. (2016a). Mapa para la Educación Especial: Comisión creada por el Gobernador presenta su informe para mejorar servicios a la población con impedimentos. Recuperado de, https://www.elnuevodia.com/noticias/ locales/nota/mapaparalaeducacionespecial-2276730/\#

López, K. (2016b). Puerto Rico reprueba en educación. Recuperado de, www.elnuevodia.com/noticias/locales/nota/anteelmundolaeducaciondepuertoric o-2269724

Mirabal, S. (2016). Augmented Storytelling Thru Student Collaboration. Denver, Colorado: Annual Conference ISTE2016.

Salcito, A. (2017). Líderes educativos y la tecnología. Corferias, Bogotá: Conferencia Virtual Educa.

Samaniego, P., Laitamo,S., Valerio, E. \& Francisco, C. (2012). Informe sobre el uso de las tecnologías de información y comunicación (TIC) en la educación para personas con discapacidad. Washington DC: UNESCO.

Santiago, J. (2017). El nivel de conocimiento que poseen los maestros de la corriente regular y los maestros del salón recurso en las escuelas públicas del municipio de Yauco sobre la co-enseñanza como estrategia educativa y cómo estos educadores implementan la misma en sus salas de clase. Tesis doctoral en Educación, Pontificia Universidad Católica de Puerto Rico, Campus de Ponce. 
Siemens, G. (2006). Knowing Knowledge. New York: Lulu, Inc.

United States of America. (2004). To amend the Assistive Technology Act of 1998 to support programs of grants to States to address the assistive technology needs of individuals with disabilities, and for other purposes. < <NOTE: Oct. 25, 2004 [H.R. 4278]. Retrieved from, https://www.gpo.gov/fdsys/pkg/PLAW108publ364/html/PLAW-108publ364.htm

Varela, G. (2018). El efecto de la adaptación curricular en el aprovechamiento académico de español en estudiantes de educación especial de tercer grado en una escuela del Municipio de Isabela, Puerto Rico. Tesis doctoral en Educación, Pontificia Universidad Católica de Puerto Rico, Campus de Ponce.

Vygotsky, L. (1978). Pensamiento y lenguaje. Madrid: Paidós

Fecha de recepción: 03/03/2019

Fecha de revisión: 16/05/2019

Fecha de aceptación: 15/07/2019 